\title{
Resveratrol Alleviates Acute Campylobacter jejuni Induced Enterocolitis in a Preclinical Murine Intervention Study
}

\author{
Markus M. Heimesaat ${ }^{1} * \mathbb{E}$, Soraya Mousavi ${ }^{1}$, Ulrike Escher ${ }^{1}$, Fábia Daniela Lobo de Sá ${ }^{2} \mathbb{D}$, \\ Elisa Peh ${ }^{3}{ }^{\oplus}$, Jörg-Dieter Schulzke ${ }^{2} \oplus$, Sophie Kittler ${ }^{3}$, Roland Bücker ${ }^{2}\left(\mathbb{D}\right.$ and Stefan Bereswill ${ }^{1}$ \\ 1 Institute of Microbiology, Infectious Diseases and Immunology, Charité-University Medicine Berlin, \\ Corporate Member of Freie Universität Berlin, Humboldt-Universität zu Berlin, and Berlin Institute of \\ Health, 12203 Berlin, Germany; soraya.mousavi@charite.de (S.M.); ulrike.escher@charite.de (U.E.); \\ stefan.bereswill@charite.de (S.B.) \\ 2 Institute of Clinical Physiology, Department of Gastroenterology, Infectious Diseases and Rheumatology, \\ Charité-Universitätsmedizin Berlin, Corporate Member of Freie Universität Berlin, Humboldt-Universität \\ zu Berlin, and Berlin Institute of Health, 12203 Berlin, Germany; \\ fabia.lobo-da-fonseca@charite.de (F.D.L.d.S.); joerg.schulzke@charite.de (J.-D.S.); \\ roland-felix.buecker@charite.de (R.B.) \\ 3 Institute for Food Quality and Food Safety, University of Veterinary Medicine Hannover, Foundation, \\ 30559 Hannover, Germany; Elisa.Peh@tiho-hannover.de (E.P.); sophie.kittler@tiho-hannover.de (S.K.) \\ * Correspondence: markus.heimesaat@charite.de; Tel.: +49-30-450524318
}

Received: 7 October 2020; Accepted: 23 November 2020; Published: 25 November 2020

\begin{abstract}
The polyphenolic compound resveratrol has been shown to exert health-beneficial properties. Given globally emerging Campylobacter infections in humans, we addressed potential anti-pathogenic, immuno-modulatory and intestinal epithelial barrier preserving properties of synthetic resveratrol in the present preclinical intervention study applying a murine acute campylobacteriosis model. Two days following peroral C. jejuni infection, secondary abiotic IL- $10^{-/-}$mice were either subjected to resveratrol or placebo via the drinking water. Whereas placebo mice suffered from acute enterocolitis at day 6 post-infection, resveratrol treatment did not only lead to improved clinical conditions, but also to less pronounced colonic epithelial apoptosis as compared to placebo application. Furthermore, C. jejuni induced innate and adaptive immune cell responses were dampened in the large intestines upon resveratrol challenge and accompanied by less colonic nitric oxide secretion in the resveratrol versus the placebo cohort. Functional analyses revealed that resveratrol treatment could effectively rescue colonic epithelial barrier function in C. jejuni infected mice. Strikingly, the disease-alleviating effects of resveratrol could additionally be found in extra-intestinal and also systemic compartments at day 6 post-infection. For the first time, our current preclinical intervention study provides evidence that peroral resveratrol treatment exerts potent disease-alleviating effects during acute experimental campylobacteriosis.
\end{abstract}

Keywords: polyphenols; resveratrol; preclinical intervention study; Campylobacter jejuni; secondary abiotic IL-10 $10^{-/}$mice; acute campylobacteriosis model; pro-inflammatory immune responses; host-pathogen interactions; intestinal epithelial barrier function

\section{Introduction}

Campylobacter infections are emerging worldwide and cause tremendous health burdens and socioeconomic costs, irrespective whether low-, middle- or high-income countries are concerned [1,2]. Among the Campylobacter genus, C. jejuni are the most prevalent species causing human illnesses [2]. 
The Gram-negative bacteria reside as commensals in the gut microbiota of, clinically, mostly unaffected warm-blooded vertebrate species including livestock such as poultry. The enteropathogens might be transferred to humans, however, following ingestion of contaminated undercooked meat or surface water $[3,4]$. Following successful passage of the stomach, adhesion to and invasion of the intestinal epithelia by C. jejuni, innate and adaptive immune cells are recruited to the colonic mucosa and lamina propria. In turn, pro-inflammatory mediator secretion leads to oxidative stress to and apoptosis of colonic epithelial cells mounting in epithelial barrier dysfunction [5,6].

Following an incubation period of between 2 and 5 days, C. jejuni induced symptoms might arise, and severity of disease ranges from relatively mild discomfort to acute campylobacteriosis characterized by fever, abdominal cramps, watery or even inflammatory and bloody diarrhea with mucous discharge depending on the enteropathogen's virulence factors and the host immune status [2,7]. Infected intestinal tissue samples display inflammatory changes including immune cell infiltrates within the mucosa and lamina propria, crypt abscesses, epithelial cell apoptosis and ulcerations [6]. In most instances, the course of disease is benign and self-limiting, and patients require, if at all, symptomatic treatment such as substitution of electrolytes. Antibiotic treatment may be required, however, in immunocompromised patients suffering from severe campylobacteriosis [2,8]. In rare instances, even post-infectious autoimmune diseases such as reactive arthritis, Guillain Barré and Miller Fisher syndromes and intestinal morbidities including chronic inflammatory bowel diseases, irritable bowel syndrome and celiac disease may develop with a latency of weeks to months post-infection (p.i.) [2,8].

Despite the emergence of human campylobacteriosis, the molecular mechanisms underlying pathogen-host interactions are only incompletely understood. In order to tackle this limitation, our group has established a murine infection and inflammation model resembling key features of severe campylobacteriosis in humans. Therefore, the commensal microbiota was depleted in IL-10-/mice by broad-spectrum antibiotic treatment in order to override the physiological colonization resistance caused by the murine gut microbiota and, hence, to facilitate stable C. jejuni infection [9]. Furthermore, since mice are approximately 10,000 times more resistant to bacterial lipooligosaccharide (LOS) and lipopolysacchride (LPS) as compared to humans [10], and il10 gene deficiency renders the murine host susceptible to pathogenic LOS and LPS, peroral C. jejuni infection of secondary abiotic IL-10 $10^{-/}$mice resulted in nonself-limiting acute enterocolitis with extra-intestinal and even systemic inflammatory manifestations within one week due to LOS-induced and Toll-like receptor-4 (TLR-4)-dependent immune responses $[9,11]$. Our murine C. jejuni infection and inflammation model has been proven reliable for testing molecules and compounds of interest regarding their anti-pathogenic and immuno-modulatory properties as shown in our recent preclinical intervention studies [12-17].

Resveratrol (3,5,4'-trihydroxy-trans-stilbene) constitutes a polyphenolic molecule that can be found in the skin and seeds of grapes, in red wine, in various fruits and in peanuts, for instance, acting as phytoalexin against pathogens such as bacteria and fungi $[18,19]$. Several in vitro and in vivo studies revealed pleiotropic health-beneficial properties of resveratrol such as anti-oxidant, anti-inflammatory, anti-microbial, anti-cancer, anti-diabetic, anti-dyslipidemic, anti-aging, cardio-protective and neuroprotective effects as reviewed in detail previously [18,19]. In our recent study we showed that resveratrol treatment could ameliorate acute small intestinal inflammation by down-regulating T helper-1 (Th-1) cell immune responses following peroral Toxoplasma gondii infection. This prompted us to test resveratrol in our present preclinical intervention study for its anti-pathogenic and immuno-modulatory effects applying another acute and fatal intestinal inflammation model of different (i.e., enteropathologic) etiology and manifestation in a different intestinal (i.e., large intestinal) compartment.

\section{Materials and Methods}

\subsection{Ethics Approval}

Mouse experiments were approved by the local ethical authorities ("Landesamt für Gesundheit und Soziales", LaGeSo, Berlin, Germany; registration numbers G0109/19, approved on 15 July 2019) 
and performed in accordance to the European Guidelines for animal welfare (2010/63/EU). The clinical conditions of mice were surveyed twice daily.

\subsection{Determination of the Minimal Inhibitory Concentration of Resveratrol}

For the assessment of the minimal inhibitory concentration (MIC) values of resveratrol, 20 C. jejuni isolates including the reference strain 81-176 used for murine infection in the present study (see below) were tested for their antimicrobial susceptibility applying the broth microdilution method. Procedures regarding inoculum density, growth medium, incubation time and conditions were performed according to the recommendations indicated in the Clinical and Laboratory Standards Institute (CLSI) document VET01-A5 [20]. Twofold serial dilutions of resveratrol ranging from 0.008 to $8.0 \mathrm{mmol} / \mathrm{L}$ $(1.78-1826 \mathrm{mg} / \mathrm{mL})$ were tested. Stock solutions were prepared in Mueller-Hinton broth and adjusted to $\mathrm{pH} 7.3$.

\subsection{Generation of Secondary Abiotic IL-10-/- Mice}

IL-10-/- mice (C57BL/6j background) were reared under specific pathogen-free conditions in the identical housing room located at the Forschungseinrichtungen für Experimentelle Medizin (Charité-University Medicine Berlin). Within an experimental semi-barrier mice were maintained in cages with filter tops under standard conditions $\left(12 \mathrm{~h}\right.$ light $/ 12$ dark cycle, $22-24{ }^{\circ} \mathrm{C}$ room temperature, $55 \pm 15 \%$ humidity) and had unlimited access to autoclaved standard food pellets (ssniff $\mathrm{R} / \mathrm{M}-\mathrm{H}$, V1534-300, Sniff, Soest, Germany). In order to assure stable gastrointestinal C. jejuni colonization by counteracting physiological colonization resistance [21], microbiota-depleted (i.e., secondary abiotic) mice were generated as stated earlier [21,22]. In brief, 3-week-old mice were challenged with an 8-week course of broad-spectrum antibiotic treatment. Therefore, five different antibiotic compounds including imipenem ( $250 \mathrm{mg} / \mathrm{L}$; Fresenius Kabi, Bad Homburg, Germany), vancomycin (500 mg/L; Hikma Pharmaceuticals, London, UK), metronidazole (1 g/L; B. Braun, Melsungen, Germany), ampicillin plus sulbactam ( $1 \mathrm{~g} / \mathrm{L}$; Dr. Friedrich Eberth Arzneimittel, Ursensollen, Germany), and ciprofloxacin $(200 \mathrm{mg} / \mathrm{L}$; Fresenius Kabi, Bad Homburg, Germany) were added to autoclaved tap water (ad libitum) as reported by us earlier [22]. The antibiotic cocktail was withdrawn three days before infection in order to assure antibiotic washout. All mice were maintained and handled under strict aseptic precautions throughout the entire experiment.

\subsection{Resveratrol Treatment}

Treatment with synthetic resveratrol (Sigma-Aldrich, München, Germany) started two days after the initial C. jejuni infection. Resveratrol was dissolved in $2 \%$ carboxy-methyl-cellulose (to a final concentration of $0.05 \%$ ) and provided to mice by adding the solution to the autoclaved tap water. The final concentration of the resveratrol solution was $300 \mathrm{mg} / \mathrm{L}$ (resulting dose of $60 \mathrm{mg}$ per $\mathrm{kg}$ body weight per day). Placebo control animals received vehicle only.

\subsection{C. jejuni Infection and Gastrointestinal Colonization}

Four-month-old sex- and age-matched littermate mice were orally challenged with $10^{9}$ viable C. jejuni strain 81-176 cells on day (d) 0 and d1 by gavage. For surveying pathogenic colonization efficacies, C. jejuni were cultured from serial dilutions of fecal samples taken during the course of infection and, additionally, of the colon, terminal ileum, duodenum and stomach lumen upon necropsy at $\mathrm{d} 6$ p.i. as reported earlier [21]. The detection limit of the C. jejuni load was 100 colony-forming units perg $(\mathrm{CFU} / \mathrm{g})$.

\subsection{Clinical Conditions}

The clinical conditions of mice were monitored before and after $C$. jejuni infection on the basis of a standardized clinical scoring system (maximum 12 points) addressing the clinical aspect/wasting 
(0: normal; 1: ruffled fur; 2: less locomotion; 3: isolation; 4: severely compromised locomotion, pre-final aspect), fecal consistency (0: formed feces; 2 : pasty feces; 4 : liquid feces) and abundance of blood in the feces (0: no blood; 2: microscopic detection of blood by the Guajac method using Haemoccult, Beckman Coulter/PCD, Germany; 4: macroscopic blood visible) as described earlier [23].

\subsection{Sampling Procedures}

At d6 p.i., mice were sacrificed by $\mathrm{CO}_{2}$ asphyxiation. Luminal samples from the colon, terminal ileum, duodenum and stomach and ex vivo biopsies from the liver, kidneys and colon as well as cardiac blood were derived under aseptic precautions. Colonic and extra-intestinal samples were taken from each mouse in parallel for subsequent immunological, immunohistopathological and microbiological analyses.

\subsection{Immunohistochemistry}

Quantitative in situ immunohistochemical analyses were done with 5\% formalin fixed and paraffin-embedded colonic tissue samples [24,25]. In brief, in order to detect apoptotic epithelial cells, macrophages/monocytes, $\mathrm{T}$ and B lymphocytes, $5 \mu \mathrm{m}$ thin colonic paraffin sections were stained with primary antibodies against cleaved caspase 3 (Asp175, Cell Signaling, Beverly, MA, USA, 1:200), F4/80 (\# 14-4801, clone BM8, eBioscience, San Diego, CA, USA, 1:50), CD3 (\#N1580, Dako, Glostrup, Denmark, 1:10), and B220 (\#14-0452-81, eBioscience; 1:200), respectively. Positively stained cells were examined by light microscopy (magnification $100 \times$ and $400 \times$ ), and for each mouse the average number of respective positively stained cells was determined within six high-power fields (HPF, $0.287 \mathrm{~mm}^{2}$, $400 \times$ magnification) by a blinded investigator.

\subsection{Measurements of Intestinal, Extra-Intestinal and Systemic Inflammatory Mediators}

After longitudinal cut, large intestinal explants were washed in phosphate-buffered saline (PBS; Gibco, Life Technologies, Loughborough, UK), and strips of colonic and liver tissues (both approximately $1 \mathrm{~cm}^{2}$ ) and one kidney (cut longitudinally) were placed in flat-bottom 24-well culture plates (Nunc, Darmstadt, Germany) containing $500 \mu \mathrm{L}$ serum-free RPMI 1640 medium (Thermo Fisher Scientific, Waltham, MA, USA) supplemented with penicillin $(100 \mathrm{U} / \mathrm{mL})$ and streptomycin $(100 \mu \mathrm{g} / \mathrm{mL}$, Biochrom, Berlin, Germany). After $18 \mathrm{~h}$ at $37^{\circ} \mathrm{C}$, respective culture supernatants as well as serum samples were tested for tumor necrosis factor- $\alpha$ (TNF- $\alpha$ ), interferon (IFN)- $\gamma$ and interleukin (IL)- 6 by the Mouse Inflammation Cytometric Bead Assay (CBA; BD Biosciences, Heidelberg, Germany) on a BD FACSCanto II flow cytometer (BD Biosciences, Heidelberg, Germany). Nitric oxide concentrations were determined by the Griess reaction as described earlier [22].

\subsection{Electrophysiological Measurements}

Ex vivo biopsies were taken from the terminal large intestines and transferred to Ussing chambers (remaining unstripped; $0.049 \mathrm{~cm}^{2}$ area). The transmural electrical resistance $\left(\mathrm{R}^{\mathrm{t}}\right)$ was measured with an automatic clamp device (CVC6, Fiebig Hard \& Software, Berlin, Germany) under voltage clamp conditions for one hour at $37^{\circ} \mathrm{C}$. The composition of the with carbogen gas $(\mathrm{pH} 7.4)$ equilibrated bathing solution was as follows: L-glutamine $(2.5 \mathrm{mmol} / \mathrm{L})$, beta-hydroxybutyric acid $(0.5 \mathrm{mmol} / \mathrm{L})$, $\mathrm{D}(+)$-glucose (10.0 mmol/L), D(+)-mannose (10.0 mmol/L), NaCl (113.6 mmol/L), KCl (5.4 mmol/L), $\mathrm{CaCl}_{2}(1.2 \mathrm{mmol} / \mathrm{L}), \mathrm{MgCl}_{2}(1.2 \mathrm{mmol} / \mathrm{L}), \mathrm{Na}_{2} \mathrm{HPO}_{4}(2.4 \mathrm{mmol} / \mathrm{L}), \mathrm{NaH}_{2} \mathrm{PO}_{4}(0.6 \mathrm{mmol} / \mathrm{L})$ and $\mathrm{NaHCO}_{3}$ $(21.0 \mathrm{mmol} / \mathrm{L})$.

\subsection{Statistical Analysis}

The significance levels (with $p$ values $\leq 0.05$ indicative for significant differences) were calculated with the following tests (applying GraphPad Prism v7, San Diego, CA, USA): Mann-Whitney test (pairwise comparison, non-normal data distribution); one-sided ANOVA test and Tukey 
post-correction (multiple comparisons; normal data distribution); the Kruskal-Wallis test and Dunn's post-correction (multiple comparisons; non-normal data distribution). Data sets were generated from four independent experiments.

\section{Results}

\subsection{Antimicrobial Properties of Resveratrol against C. jejuni In Vitro}

First, we investigated potential antimicrobial effects of resveratrol directed against C. jejuni. In vitro studies with $20 \mathrm{C}$. jejuni isolates including the reference strain 81-176 revealed a resveratrol $\mathrm{MIC}_{90}$ value of $228.25 \mathrm{mg} / \mathrm{L}(1.0 \mathrm{mmol} / \mathrm{L})$ with MICs ranging from $57.1 \mathrm{mg} / \mathrm{L}(0.25 \mathrm{mmol} / \mathrm{L})$ to $456.5 \mathrm{mg} / \mathrm{L}$ $(2.0 \mathrm{mmol} / \mathrm{L})$ (Supplementary Figure S1), whereas the C. jejuni strain 81-176 yielded an MIC value of $456.5 \mathrm{mg} / \mathrm{L}(2.0 \mathrm{mmol} / \mathrm{L})$.

\subsection{Gastrointestinal Pathogen Loads Following Resveratrol Treatment of Mice Suffering from C. jejuni Induced Enterocolitis}

Following peroral infection with $10^{9}$ viable C. jejuni strain $81-176$ cells on $\mathrm{d} 0$ and $\mathrm{d} 1$, the pathogen could stably establish within the intestinal tract of secondary abiotic IL-10 ${ }^{-/}$mice from either cohort as indicated by median fecal loads of approximately $10^{9}$ CFU per g (Supplementary Figure S2). On d3, d5 and d6 p.i., however, fecal C. jejuni loads were slightly lower (up to one log order of magnitude) in resveratrol- as compared to placebo-treated mice $(p<0.05-0.001$; Supplementary Figure S2). Furthermore, upon necropsy we surveyed the pathogen densities alongside the gastrointestinal tract. Whereas the stomach, duodenum and ileum of resveratrol- and placebo-treated mice harbored comparable pathogen numbers, one order of magnitude lower $C$. jejuni counts could be assessed in luminal samples taken from the colon in the former as compared to the latter on d6 p.i. $(p<0.001$; Figure 1). Hence, resveratrol-treated mice harbored slightly lower $C$. jejuni cell counts in their large intestines as compared to placebo controls.

\section{C. jejuni - Gastrointestinal Tract}

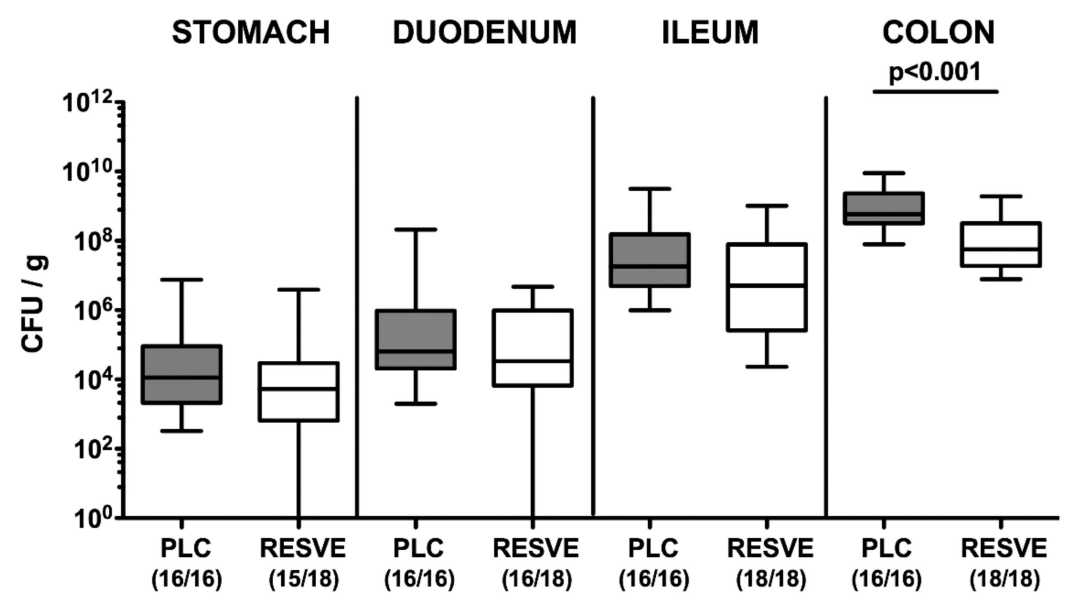

Figure 1. Gastrointestinal C. jejuni loads following resveratrol treatment of infected mice suffering from acute enterocolitis. Starting two days after initial peroral infection with C. jejuni strain 81-176 on day (d) 0 and $\mathrm{d} 1$, secondary abiotic IL-10-/- mice were treated with resveratrol (RESVE; white boxes) or placebo (PLC; grey boxes) via the drinking water. On d6 post-infection, C. jejuni loads were quantitatively assessed in distinct parts of the gastrointestinal tract by culture (in colony-forming units (CFU) per $g$ feces). Box plots represent the 75th and 25th percentiles of the median (black bar inside the boxes). Total range, significance levels ( $p$ values as determined by the Mann-Whitney test) and the number of culture-positive mice out of the total number of analyzed animals (in parentheses) are given. Data were pooled from three independent experiments. 


\subsection{Clinical Conditions of Resveratrol-Treated Mice Suffering from C. jejuni Induced Enterocolitis}

We further assessed the clinical outcome of $C$. jejuni infection over time by applying a standardized scoring system by which wasting, severity of diarrhea and abundance of fecal blood could be quantified. Within six days, C. jejuni infected mice from the placebo cohort developed acute enterocolitis as indicated by wasting and bloody diarrhea, whereas in resveratrol-treated mice clinical signs were less pronounced as early as d5 p.i. $(p<0.01$; Supplementary Figure S3). At the end of the observation period, median clinical scores of 12 and 4 could be determined in the placebo and resveratrol cohorts, respectively $(p<0.01$; Figure 2A). Notably, the standard deviation in the resveratrol group was relatively high, given that some mice were suffering from full blown disease, whereas others displayed only mild or even no clinical signs of $C$. jejuni infection at all (Figure 2A). Hence, resveratrol treatment resulted in a better clinical outcome of $C$. jejuni induced disease.

\section{A Clinical Conditions}

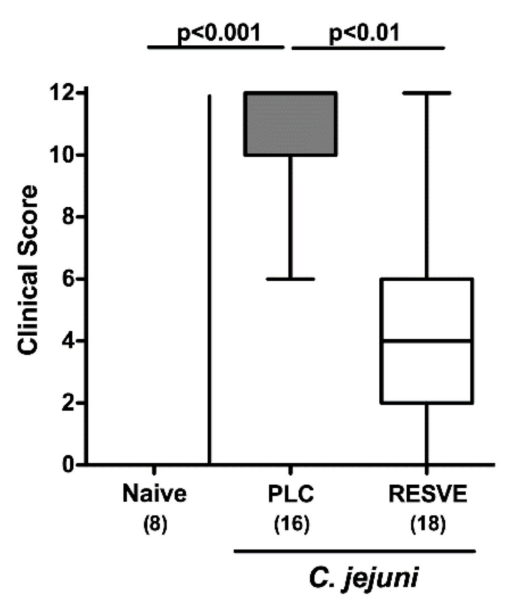

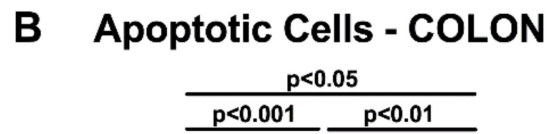

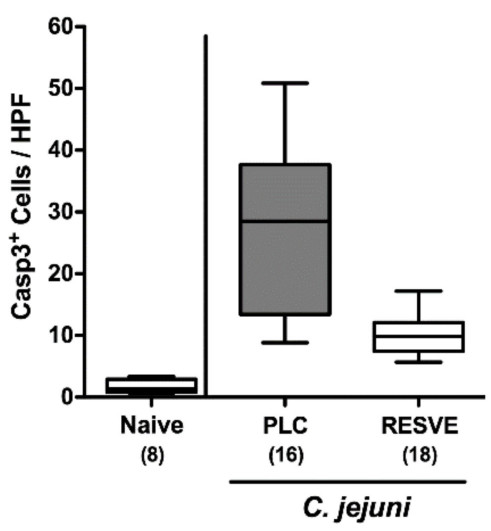

Figure 2. Clinical conditions and microscopic inflammatory responses following resveratrol treatment of infected mice suffering from acute enterocolitis. Starting two days after initial peroral infection with C. jejuni strain 81-176 on $\mathrm{d} 0$ and $\mathrm{d} 1$, secondary abiotic IL-10 ${ }^{-/-}$mice were treated with resveratrol (RESVE; white boxes) or placebo (PLC; grey boxes) via the drinking water. On d6 post-infection, (A) clinical conditions were quantitatively assessed applying a clinical scoring system. Furthermore, (B) apoptotic cells (cleaved caspase $3^{+}$, Casp $3^{+}$) were assessed microscopically from six high-power fields (HPF, 400× magnification) per animal in immunohistochemically stained colonic paraffin sections. Naive mice served as untreated and uninfected controls. Box plots represent the 75th and 25th percentiles of the median (black bar inside the boxes). Total range, significance levels ( $p$ values as determined by the Kruskal-Wallis test followed by Dunn's correction) and numbers of mice (in parentheses) are given.

Data were pooled from three independent experiments.

3.4. Apoptotic Responses in Large Intestinal Epithelia of Resveratrol-Treated Mice Suffering from C. jejuni Induced Enterocolitis

Given that apoptosis is regarded a reliable parameter for the grading of intestinal inflammation [21], we quantitatively assessed apoptotic cells in colonic epithelia applying in situ immunohistochemistry. In fact, $C$. jejuni infection was associated with multifold increases in cleaved caspase $3^{+}$cell numbers in colonic epithelia ( $p<0.05-0.001$ versus naive; Figure 2B, Supplementary Figure S4A). These increases, however, were far less pronounced following resveratrol as compared to placebo treatment at $\mathrm{d} 6$ p.i. ( $p<0.01$; Figure 2B, Supplementary Figure S4A). Hence, ameliorated gross C. jejuni induced disease was accompanied by dampened apoptotic cell responses in the large intestinal epithelia upon resveratrol treatment. 
3.5. Inflammatory Immune Responses in the Large Intestines of Resveratrol-Treated Mice Suffering from C. jejuni Induced Enterocolitis

We further addressed whether resveratrol treatment was associated with less pronounced C. jejuni induced immune responses. We therefore quantified distinct innate as well as adaptive immune cell populations following immunohistochemical stainings of colonic paraffin sections with defined antibodies. At $\mathrm{d} 6$ following C. jejuni infection, markedly increased numbers of innate immune cell subsets such as $\mathrm{F} 4 / 80^{+}$macrophages and monocytes as well as of adaptive immune cell populations including $\mathrm{CD}^{+} \mathrm{T}$ lymphocytes and $\mathrm{B} 220^{+} \mathrm{B}$ cells could be observed in the colonic mucosa and lamina propria $(p<0.05-0.001$; Figure 3A-C, Supplementary Figure S4B-D). In resveratrol-treated mice, however, colonic numbers of either immune cell subsets were lower at $\mathrm{d} 6 \mathrm{p}$.i. as compared to placebo controls $(p<0.05-0.01$; Figure 3A-C, Supplementary Figure S4B-D). Less pronounced increases in large intestinal mucosal immune cells were paralleled by less $C$. jejuni induced secretion of nitric oxide in colonic ex vivo biopsies obtained from resveratrol- as compared to placebo-treated mice at $\mathrm{d} 6$ p.i. $(p<0.05$; Figure 3D). Hence, resveratrol treatment resulted in less pronounced $C$. jejuni induced pro-inflammatory immune responses in the large intestinal tract.

A Macrophages/Monocytes

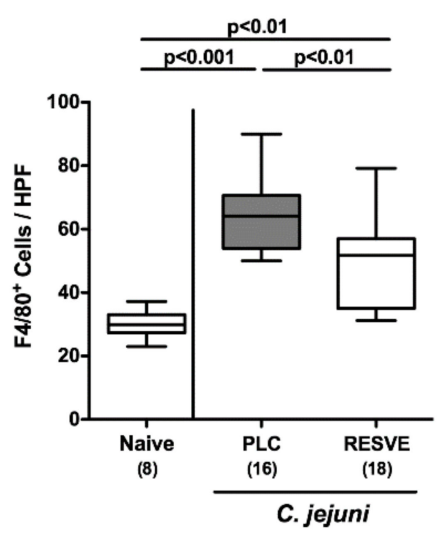

C
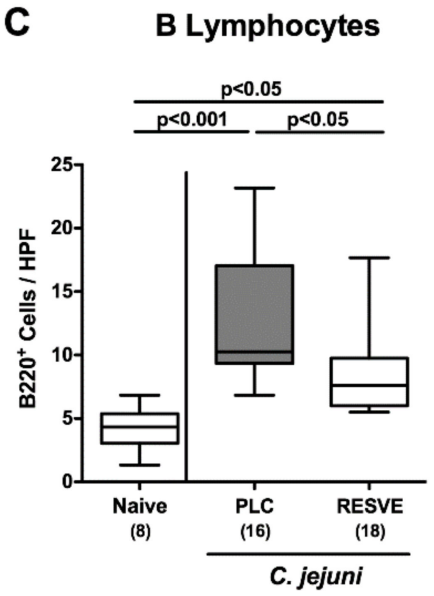

\section{B T Lymphocytes}

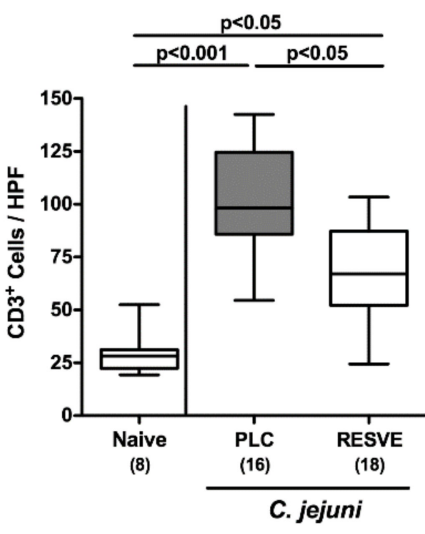

D Nitric Oxide

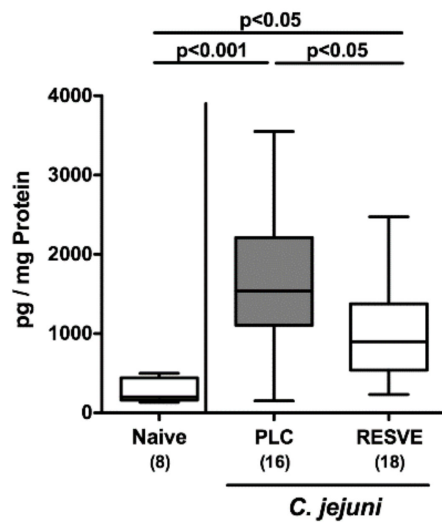

Figure 3. Inflammatory immune responses upon resveratrol treatment of infected mice suffering from acute enterocolitis. Starting two days after initial peroral infection with C. jejuni strain 81-176 on d0 and $\mathrm{d} 1$, secondary abiotic IL-10-/- mice were treated with resveratrol (RESVE; white boxes) or placebo (PLC; grey boxes) via the drinking water. On d6 post-infection, (A) macrophages and monocytes $\left(\mathrm{F} 4 / 80^{+}\right)$, (B) T lymphocytes $\left(\mathrm{CD}^{+}\right)$and $(\mathrm{C})$ B lymphocytes $\left(\mathrm{B} 220^{+}\right)$were assessed microscopically from six high-power 
fields (HPF, 400× magnification) per animal in immunohistochemically stained colonic paraffin sections. Furthermore, (D) nitric oxide concentrations were measured in colonic ex vivo biopsies. Naive mice served as untreated and uninfected controls. Box plots represent the 75th and 25th percentiles of the median (black bar inside the boxes). Total range, significance levels ( $p$ values as determined by the one-sided ANOVA test followed by Tukey correction or by the Kruskal-Wallis test followed by Dunn's correction) and numbers of mice (in parentheses) are given. Data were pooled from three independent experiments.

\subsection{Colonic Epithelial Barrier Function of Resveratrol-Treated Mice Suffering from C. jejuni Induced} Enterocolitis

We further addressed whether resveratrol treatment could rescue epithelial barrier function following C. jejuni infection. Therefore, we performed electrophysiological resistance measurements of colonic ex vivo biopsies in the Ussing chamber. In fact, transmural resistances were lower in the large intestines derived from placebo, but not resveratrol-treated mice, at d6 p.i. as compared to naive controls ( $p<0.05$ versus naive, $p<0.001$ versus resveratrol; Figure 4 ). Hence, resveratrol treatment could effectively rescue colonic epithelial barrier function following $C$. jejuni infection.

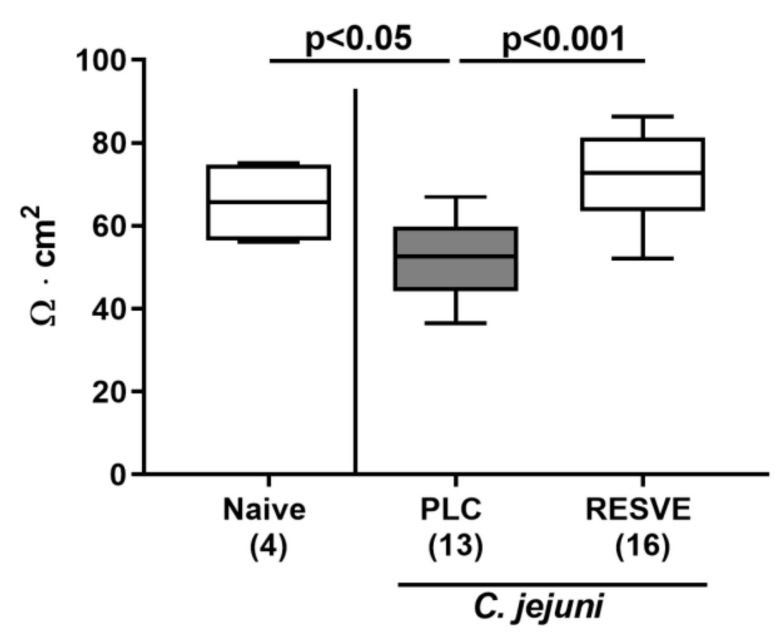

Figure 4. Colonic transmural electrical resistance following resveratrol treatment of $C$. jejuni infected mice. Starting two days after initial peroral infection with C. jejuni strain 81-176 on d0 and d1, secondary abiotic IL-10 ${ }^{-/-}$mice were treated with resveratrol (RESVE; white boxes) or placebo (PLC; grey boxes) via the drinking water. On d6 post-infection, the transmural electrical resistance (Rt) of the distal colon was measured in Ussing chambers (in $\Omega \cdot \mathrm{cm}^{2}$ ). Naive mice served as untreated and uninfected controls. Box plots represent the 75th and 25th percentiles of the median (black bar inside the boxes). Total range, significance levels ( $p$ values as determined by the one-sided ANOVA test followed by Tukey correction) and numbers of mice (in parentheses) are given. Data were pooled from two independent experiments.

3.7. Extra-Intestinal Including Systemic Pro-Inflammatory Responses in Resveratrol-Treated Mice Suffering from C. jejuni Induced Enterocolitis

Next, we addressed whether the disease-alleviating effects of resveratrol treatment were restricted to the intestinal tract or also effective in extra-intestinal compartments. Therefore, we measured pro-inflammatory mediators in ex vivo biopsies taken from kidneys and the liver. In fact, increased nitric oxide, TNF- $\alpha$ and IFN- $\gamma$ concentrations could exclusively be assessed in the kidneys of $C$. jejuni infected mice from the placebo cohort ( $p<0.05-0.001$ versus naive; Figure $5 \mathrm{~A}-\mathrm{C})$, which also held true for hepatic IFN- $\gamma$ levels ( $p<0.05$; Figure 5D). 
A Nitric Oxide - KIDNEY

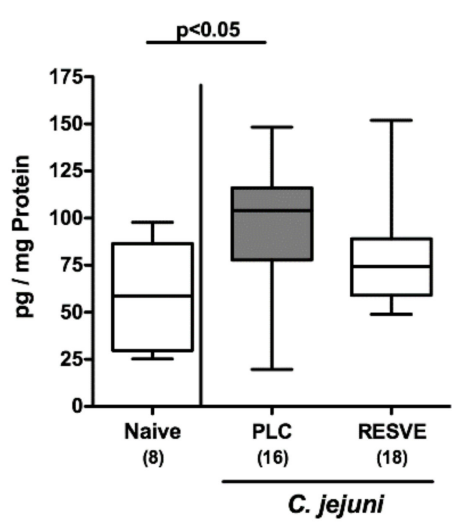

C

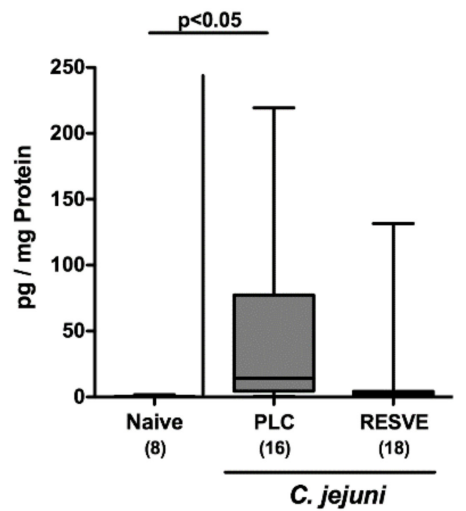

B TNF - KIDNEY

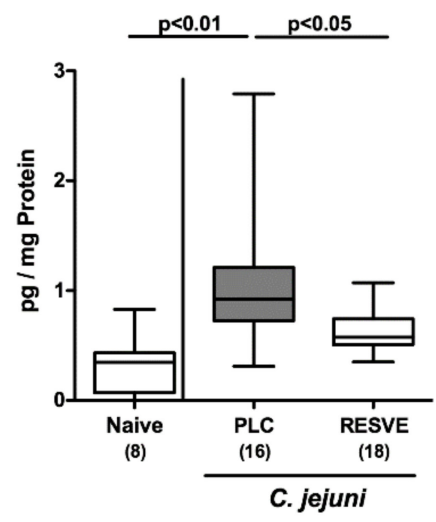

D IFN- $\gamma$ - LIVER

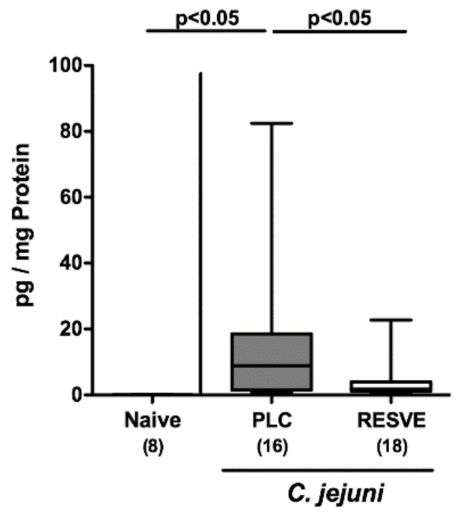

Figure 5. Inflammatory mediator secretion in extra-intestinal compartments following resveratrol treatment of infected mice suffering from acute enterocolitis. Starting two days after initial peroral infection with $C$. jejuni strain $81-176$ on $\mathrm{d} 0$ and $\mathrm{d} 1$, secondary abiotic IL-10 ${ }^{-/}$mice were treated with resveratrol (RESVE; white boxes) or placebo (PLC; grey boxes) via the drinking water. On d6 post-infection, extra-intestinal concentrations of (A) nitric oxide, (B) TNF- $\alpha$ and (C,D) IFN- $\gamma$ were measured in ex vivo biopsies derived from kidneys (A-C) and the liver (D). Naive mice served as untreated and uninfected controls. Box plots represent the 75th and 25th percentiles of the median (black bar inside the boxes). Total range, significance levels ( $p$ values as determined by the one-sided ANOVA test followed by Tukey correction) and numbers of mice (in parentheses) are given. Data were pooled from three independent experiments.

Strikingly, resveratrol-mediated dampening of C. jejuni induced disease could also be observed systemically given that serum IL-6 concentrations were lower following resveratrol as compared to placebo treatment at d6 p.i. $(p<0.05$; Figure 6$)$, whereas a trend towards lower TNF- $\alpha$ concentrations could be observed in sera of the former as compared to the latter (not significant due to high standard deviations; Figure 6B). Hence, resveratrol treatment not only alleviated intestinal responses, but also extra-intestinal including systemic C. jejuni induced inflammatory responses. 
A

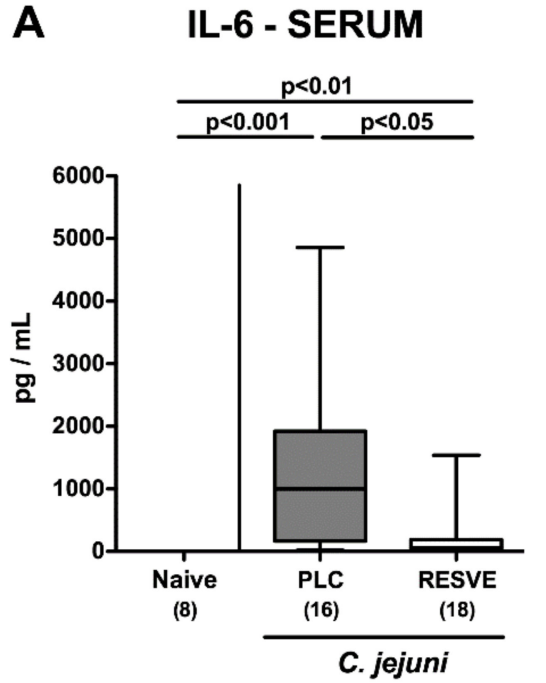

B

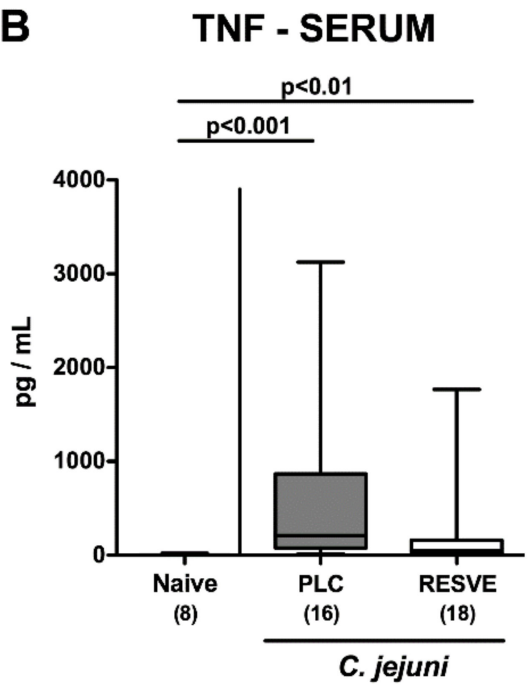

Figure 6. Systemic pro-inflammatory mediator secretion following resveratrol treatment of infected mice suffering from acute enterocolitis. Starting four days prior peroral infection with C. jejuni 81-176 strain on $\mathrm{d} 0$ and $\mathrm{d} 1$, secondary abiotic IL-10 ${ }^{-/-}$mice were treated with resveratrol (RESVE; white boxes) or placebo (PLC; grey boxes) via the drinking water. On d6 post-infection, serum concentrations of (A) IL-6 and (B) TNF- $\alpha$ were measured. Naive mice served as untreated and uninfected controls. Box plots represent the 75th and 25th percentiles of the median (black bar inside the boxes). Total range, significance levels ( $p$ values; as determined by the Kruskal-Wallis test followed by Dunn's correction) and numbers of mice (in parentheses) are given. Data were pooled from three independent experiments.

\section{Discussion}

In our present preclinical intervention study we show, for the first time, that synthetic resveratrol application in the here-applied murine acute campylobacteriosis model (i) slightly lowered large intestinal luminal C. jejuni loads, (ii) resulted in a better clinical outcome that was accompanied by (iii) less distinct apoptosis of colonic epithelial cells and (iv) less pronounced pro-inflammatory immune responses in intestinal, extra-intestinal and, strikingly, systemic compartments and, furthermore, (v) in preserved colonic epithelial barrier function.

The observed disease-alleviating effects of resveratrol treatment were rather due to the amplified immune response than antimicrobial effects. Resveratrol has been shown to exert bacteriostatic properties against both Gram-positive and Gram-negative bacteria such as Staphylococcus aureus and Escherichia coli, respectively [26,27]. However, the underlying mechanisms are not yet fully understood. Changes in bacterial DNA content and disruption of bacterial membrane formation have been proposed, for instance [26-28]. Furthermore, resveratrol could effectively inhibit the adhesion of enteropathogens such as Salmonella enterica subspecies enterica serovar Typhimurium and enteropathogenic E. coli to colonic epithelial cell lines [29], whereas the polyphenolic compound could also antagonize the adhesive properties of $C$. jejuni to both biotic and abiotic surface structures [30]. Moreover, resveratrol prevented biofilm formation by C. jejuni, E. coli and Aliarcobacter species even when applied in concentrations below the MIC [31]. A slight reduction in the intestinal pathogen burden of one order of magnitude was observed in our present study, where the concentration of the applied resveratrol solution (300 mg/L) was slightly higher than the resveratrol $\mathrm{MIC}_{90}$ values (i.e., $228.25 \mathrm{mg} / \mathrm{L}$ ) determined for 20 C. jejuni isolates, but below the resveratrol MIC of the C. jejuni strain 81-176 determined in vitro. Further, one needs to take into consideration that, following peroral application, resveratrol is rapidly metabolized in the body, and despite an absorption rate reaching $70 \%$, the oral bioavailability needs to be considered as rather low $[18,32]$. Given that peroral resveratrol is also metabolized by the resident gut microbiota, it is unknown whether the observed effects are due to resveratrol alone and/or its metabolites [33]. 
The better clinical outcome of $C$. jejuni induced acute disease as indicated by less severe wasting and bloody diarrhea following resveratrol versus placebo application was accompanied by less pronounced colonic epithelial apoptosis and less colonic, extra-intestinal and systemic secretion of pro-inflammatory mediators. In support, a recent study applying a rabbit model of acute pharyngitis revealed that the anti-inflammatory effects of resveratrol were due to down-regulated caspase-3 expression and decreased IL-6 and TNF- $\alpha$ serum levels [34]. Resveratrol could effectively modulate both innate and adaptive immune responses upon C. jejuni infection, given that numbers in macrophages and monocytes as well as of $\mathrm{T}$ and $\mathrm{B}$ lymphocytes in the colonic mucosa and lamina propria were lower following resveratrol as compared to placebo application. These results are well in line with a previous in vitro study demonstrating that resveratrol impaired GM-CSF production [35] mounting in decreased differentiation, activation and survival of pro-inflammatory macrophages [36]. Furthermore, resveratrol application resulted in a dampened production of IFN- $\gamma$ and TNF- $\alpha$ by activated macrophages and $\mathrm{T}$ lymphocytes in vitro $[37,38]$. In line, our group recently demonstrated that peroral resveratrol treatment of mice led to diminished $\mathrm{T}$ cell responses upon Toxoplasma gondii infection and subsequently prevented the animals from developing peracute ileitis [39]. In our present study, C. jejuni infected mice from the resveratrol cohort did not only exhibit decreased $T$ cell, but also $B$ cell numbers in their colonic mucosa and lamina propria. In support, resveratrol was shown to inhibit B cell proliferation in a murine model of systemic lupus erythematosus [40]. The diminished innate and adaptive immune cell responses upon resveratrol treatment of $C$. jejuni infected mice here were accompanied by reduced colonic secretion of nitric oxide, hence diminishing oxidative stress to the large intestinal epithelia and resulting in a preserved epithelial barrier function. Moreover, the reduction in epithelial apoptosis can provide an argument for a direct improvement in the transmural resistance in the colon of the infected and resveratrol-treated mice, since it was shown in vitro that apoptosis induction by C. jejuni is barrier-relevant and has the main impact on the epithelium in this context, besides tight junction changes [5]. The inhibition of apoptosis induction could restore barrier function in C. jejuni infection [5]. In support, resveratrol was shown to inhibit nitric oxide secretion from LPS-stimulated macrophages in vitro [41] and to reduce oxidative stress and inflammation by dampening the expression of the inducible nitric oxide synthase (iNOS) in vivo [42]. Moreover, resveratrol application to broilers could effectively ameliorate stress-induced impairment of intestinal barrier function [43].

Notably, in our intervention study resveratrol treatment did not only alleviate pro-inflammatory immune responses in the intestinal tract, but also in extra-intestinal and even systemic compartments of C. jejuni infected mice given attenuated pro-inflammatory cytokine secretion in the kidneys, the liver and the systemic blood stream. Our results are supported by multiple studies reporting potent anti-inflammatory effects of resveratrol in the kidneys, as shown in rat mesangial cell and human renal epithelial cells in vitro, for instance, and in several renal in vivo inflammation models as recently reviewed by Den Hartogh and colleagues [44]. Furthermore, anti-inflammatory and anti-oxidant effects of resveratrol were described in hepatic ischemic reperfusion injuries in diabetic rats [45] and in experimental as well as in clinical non-alcoholic fatty liver disease [46,47]. In addition, pronounced systemic disease-alleviating effects of resveratrol could be assessed in experimental systemic lupus erythematosus [40].

It is well known that the immunopathological sequelae during campylobacteriosis are caused by TLR-4-dependent host immune responses that are induced by LOS derived from the bacterial cell wall [11,48]. Since resveratrol has been shown to interact with TLR-4 [49] and to alleviate inflammation-induced cell damage in vascular endothelial cells by suppressing the TLR-4-dependent nuclear factor (NF)-кB signaling pathway [50], this suggests another health-beneficial mode of resveratrol action when applied in murine campylobacteriosis. 


\section{Conclusions}

In conclusion, our preclinical intervention study provides strong evidence, for the first time, that synthetic resveratrol might be a promising candidate molecule to treat patients suffering from severe campylobacteriosis.

Long-term clinical trials revealed that resveratrol was well-tolerated by the individuals with doses of up to $5 \mathrm{~g}$ per day [51,52] and might therefore be considered as safe. Further studies either with resveratrol alone or in combination with other polyphenolic and/or non-polyphenolic compounds need to be performed in the future.

Supplementary Materials: The following are available online at http:/www.mdpi.com/2076-2607/8/12/1858/s1, Figure S1: Distribution of minimal inhibitory concentrations (MICs) of resveratrol among 20 C. jejuni isolates (in $\mathrm{mmol} / \mathrm{L}$ and $\mathrm{mg} / \mathrm{L}$ ). The resveratrol $\mathrm{MIC}_{90}$ value indicates the resveratrol concentration resulting in inhibition growth of $90 \%$ of the tested C. jejuni isolates; Figure S2. Fecal C. jejuni shedding over time following resveratrol treatment of infected mice suffering from acute enterocolitis; Figure S3. Clinical conditions over time following resveratrol treatment of infected mice suffering from acute enterocolitis; Figure S4. Representative photomicrographs illustrating colonic apoptotic and immune cell responses following resveratrol treatment of infected mice suffering from acute enterocolitis.

Author Contributions: M.M.H.: Designed and performed experiments, analyzed data, wrote paper; S.M.: Performed experiments, analyzed data, co-edited paper; U.E.: Performed experiments; F.D.L.d.S.: Performed experiments, analyzed data, co-edited paper; E.P.: Performed experiments, analyzed data, co-edited paper; J.-D.S.: Critically discussed results, co-edited paper; S.K.: Performed experiments, critically discussed results, co-edited paper; R.B.: Provided advice in experimental design, critically discussed results, co-edited paper; S.B.: Provided advice in experimental design, critically discussed results, co-edited paper. All authors have read and agreed to the published version of the manuscript.

Funding: This work was supported by the German Federal Ministries of Education and Research (BMBF) in frame of the zoonoses research consortium PAC-Campylobacter to S.M., S.B. and M.M.H. (IP7/01KI1725D), to J.-D.S and R.B. (IP8/01KI1725D), and to S.K. (IP5/01K11725C) and by the German Federal Ministries of Economy and Energy to S.B. and M.M.H. (ZIM; ZF4117904 AJ8). The funders had no role in study design, data collection and analysis, decision to publish or preparation of the manuscript.

Acknowledgments: The excellent technical assistance by Alexandra Bittroff-Leben, Ines Puschendorf, Ulrike Fiebiger, Anna-Maria Schmidt, Gernot Reifenberger, and the staff of the animal research facility at CharitéUniversity Medicine Berlin are gratefully acknowledged. We thank Anja A. Kühl (Department of Medicine I for Gastroenterology, Infectious Diseases and Rheumatology/Research Center ImmunoSciences (RCIS), Charité-Universitätsmedizin Berlin) for taking representative photomicropgraphs of immunohistochemically stained paraffin sections. We appreciate support from the German Research Foundation (DFG) and from the Open Access Publication Fund of Charité-Universitätsmedizin Berlin.

Conflicts of Interest: The authors declare no conflict of interest.

$\begin{array}{ll}\text { Abbreviations } \\ \text { CBA } & \text { cytometric bead array } \\ \text { CFU } & \text { colony forming units } \\ \text { CLSI } & \text { Clinical and Laboratory Standards Institute } \\ \text { HPF } & \text { high-power fields } \\ \text { IFN } & \text { interferon } \\ \text { IL } & \text { interleukin } \\ \text { iNOS } & \text { inducible nitric oxide synthase } \\ \text { LOS } & \text { lipooligosaccharide } \\ \text { LPS } & \text { lipopolysaccharide } \\ \text { MIC } & \text { minimal inhibitory concentration } \\ \text { NF } & \text { nuclear factor } \\ \text { PBS } & \text { phosphate-buffered saline } \\ \text { p.i. } & \text { post-infection } \\ \text { R } & \text { transmural electrical resistance } \\ \text { TLR } & \text { Toll-like receptor } \\ \text { TNF- } \alpha & \text { tumor necrosis factor- } \alpha \\ \text { Th-1 cells } & \text { T helper-1 cells }\end{array}$




\section{References}

1. WHO. Campylobacter. Available online: https://www.who.int/news-room/fact-sheets/detail/campylobacter (accessed on 4 June 2020).

2. Backert, S.; Tegtmeyer, N.; Cróinín, T.Ó.; Boehm, M.; Heimesaat, M.M. Chapter 1-Human campylobacteriosis. In Campylobacter; Klein, G., Ed.; Academic Press: Cambridge, MA, USA, 2017; pp. 1-25. [CrossRef]

3. Skirrow, M. Campylobacter enteritis: A "new" disease. Br. Med. J. 1977, 2, 9-11. [CrossRef]

4. Ellis-Iversen, J.; Ridley, A.; Morris, V.; Sowa, A.; Harris, J.; Atterbury, R.; Sparks, N.; Allen, V. Persistent environmental reservoirs on farms as risk factors for Campylobacter in commercial poultry. Epidemiol. Infect. 2012, 140, 916-924. [CrossRef]

5. Butkevych, E.; de Sá, F.D.L.; Nattramilarasu, P.K.; Bücker, R. Contribution of Epithelial Apoptosis and Subepithelial Immune Responses in Campylobacter jejuni-Induced Barrier Disruption. Front. Microbiol. 2020, 11. [CrossRef]

6. Young, K.T.; Davis, L.M.; Dirita, V.J. Campylobacter jejuni: Molecular biology and pathogenesis. Nat. Rev. Microbiol. 2007, 5, 665-679. [CrossRef]

7. Kist, M.; Bereswill, S. Campylobacter jejuni . Contrib. Microbiol. 2001, 8, 150-165. [CrossRef] [PubMed]

8. Kaakoush, N.O.; Castano-Rodriguez, N.; Mitchell, H.M.; Man, S.M. Global Epidemiology of Campylobacter Infection. Clin. Microbiol. Rev. 2015, 28, 687-720. [CrossRef] [PubMed]

9. Haag, L.M.; Fischer, A.; Otto, B.; Plickert, R.; Kuhl, A.A.; Gobel, U.B.; Bereswill, S.; Heimesaat, M.M. Campylobacter jejuni induces acute enterocolitis in gnotobiotic IL-10-/- mice via Toll-like-receptor-2 and -4 signaling. PLoS ONE 2012, 7, e40761. [CrossRef] [PubMed]

10. Taveira da Silva, A.M.; Kaulbach, H.C.; Chuidian, F.S.; Lambert, D.R.; Suffredini, A.F.; Danner, R.L. Brief report: Shock and multiple-organ dysfunction after self-administration of Salmonella endotoxin. N. Engl. J. Med. 1993, 328, 1457-1460. [CrossRef] [PubMed]

11. Mousavi, S.; Bereswill, S.; Heimesaat, M.M. Novel Clinical Campylobacter jejuni Infection Models Based on Sensitization of Mice to Lipooligosaccharide, a Major Bacterial Factor Triggering Innate Immune Responses in Human Campylobacteriosis. Microorganisms 2020, 8, 482. [CrossRef]

12. Mousavi, S.; Schmidt, A.M.; Escher, U.; Kittler, S.; Kehrenberg, C.; Thunhorst, E.; Bereswill, S.; Heimesaat, M.M. Carvacrol ameliorates acute campylobacteriosis in a clinical murine infection model. Gut. Pathog. 2020, 12, 2. [CrossRef]

13. Mousavi, S.; Escher, U.; Thunhorst, E.; Kittler, S.; Kehrenberg, C.; Bereswill, S.; Heimesaat, M.M. Vitamin C alleviates acute enterocolitis in Campylobacter jejuni infected mice. Sci. Rep. 2020, 10, 2921. [CrossRef] [PubMed]

14. Lobo de Sá, F.D.; Butkevych, E.; Nattramilarasu, P.K.; Fromm, A.; Mousavi, S.; Moos, V.; Golz, J.C.; Stingl, K.; Kittler, S.; Seinige, D. Curcumin mitigates immune-induced epithelial barrier dysfunction by Campylobacter jejuni. Int. J. Mol. Sci. 2019, 20, 4830. [CrossRef] [PubMed]

15. Mousavi, S.; Lobo de Sa, F.D.; Schulzke, J.D.; Bucker, R.; Bereswill, S.; Heimesaat, M.M. Vitamin D in Acute Campylobacteriosis-Results From an Intervention Study Applying a Clinical Campylobacter jejuni Induced Enterocolitis Model. Front. Immunol. 2019, 10, 2094. [CrossRef] [PubMed]

16. Heimesaat, M.M.; Mousavi, S.; Klove, S.; Genger, C.; Weschka, D.; Giladi, E.; Bereswill, S.; Gozes, I. Immune-modulatory Properties of the Octapeptide NAP in Campylobacter jejuni Infected Mice Suffering from Acute Enterocolitis. Microorganisms 2020, 8, 802. [CrossRef] [PubMed]

17. Heimesaat, M.M.; Mousavi, S.; Klove, S.; Genger, C.; Weschka, D.; Tamas, A.; Reglodi, D.; Bereswill, S. Pituitary Adenylate Cyclase-Activating Polypeptide Alleviates Intestinal, Extra-Intestinal and Systemic Inflammatory Responses during Acute Campylobacter jejuni-induced Enterocolitis in Mice. Pathogens 2020, 9, 805. [CrossRef] [PubMed]

18. Salehi, B.; Mishra, A.P.; Nigam, M.; Sener, B.; Kilic, M.; Sharifi-Rad, M.; Fokou, P.V.T.; Martins, N.; Sharifi-Rad, J. Resveratrol: A double-edged sword in health benefits. Biomedicines 2018, 6, 91. [CrossRef] [PubMed]

19. Malaguarnera, L. Influence of resveratrol on the immune response. Nutrients 2019, 11, 946. [CrossRef] [PubMed]

20. CLSI. VET01-A5: Performance Standards for Antimicrobial Disk and Dilution Susceptibility Tests for Bacteria Isolated from Animals, Approved Standard, 5th ed.; Clinical and Laboratory Standards Institute: Wayne, PA, USA, 2018; Volume 34. 
21. Bereswill, S.; Fischer, A.; Plickert, R.; Haag, L.M.; Otto, B.; Kuhl, A.A.; Dasti, J.I.; Zautner, A.E.; Munoz, M.; Loddenkemper, C.; et al. Novel murine infection models provide deep insights into the "menage a trois" of Campylobacter jejuni, microbiota and host innate immunity. PLoS ONE 2011, 6, e20953. [CrossRef]

22. Heimesaat, M.M.; Bereswill, S.; Fischer, A.; Fuchs, D.; Struck, D.; Niebergall, J.; Jahn, H.K.; Dunay, I.R.; Moter, A.; Gescher, D.M.; et al. Gram-negative bacteria aggravate murine small intestinal Th1-type immunopathology following oral infection with Toxoplasma gondii. J. Immunol. 2006, 177, 8785-8795. [CrossRef]

23. Heimesaat, M.M.; Alutis, M.; Grundmann, U.; Fischer, A.; Tegtmeyer, N.; Bohm, M.; Kuhl, A.A.; Gobel, U.B.; Backert, S.; Bereswill, S. The role of serine protease HtrA in acute ulcerative enterocolitis and extra-intestinal immune responses during Campylobacter jejuni infection of gnotobiotic IL-10 deficient mice. Front. Cell Infect. Microbiol. 2014, 4, 77. [CrossRef]

24. Heimesaat, M.M.; Nogai, A.; Bereswill, S.; Plickert, R.; Fischer, A.; Loddenkemper, C.; Steinhoff, U.; Tchaptchet, S.; Thiel, E.; Freudenberg, M.A.; et al. MyD88/TLR9 mediated immunopathology and gut microbiota dynamics in a novel murine model of intestinal graft-versus-host disease. Gut 2010, 59, 1079-1087. [CrossRef] [PubMed]

25. Heimesaat, M.M.; Giladi, E.; Kuhl, A.A.; Bereswill, S.; Gozes, I. The octapetide NAP alleviates intestinal and extra-intestinal anti-inflammatory sequelae of acute experimental colitis. Peptides 2018, 101, 1-9. [CrossRef] [PubMed]

26. Paulo, L.; Ferreira, S.; Gallardo, E.; Queiroz, J.A.; Domingues, F. Antimicrobial activity and effects of resveratrol on human pathogenic bacteria. World J. Microbiol. Biotechnol. 2010, 26, 1533-1538. [CrossRef]

27. Hwang, D.; Lim, Y. Resveratrol antibacterial activity against Escherichia coli is mediated by Z-ring formation inhibition via suppression of FtsZ expression. Sci. Rep. 2015, 5, 10029. [CrossRef]

28. Lee, W.; Lee, D.G. Resveratrol induces membrane and DNA disruption via pro-oxidant activity against Salmonella typhimurium. Biochem. Biophys. Res. Commun. 2017, 489, 228-234. [CrossRef]

29. Selma, M.V.; Larrosa, M.; Beltrán, D.; Lucas, R.; Morales, J.C.; Tomás-Barberán, F.; Espín, J.C. Resveratrol and some glucosyl, glucosylacyl, and glucuronide derivatives reduce Escherichia coli O157:H7, Salmonella Typhimurium, and Listeria monocytogenes Scott A adhesion to colonic epithelial cell lines. J. Agric. Food Chem. 2012, 60, 7367-7374. [CrossRef]

30. Klančnik, A.; Šikić Pogačar, M.; Trošt, K.; Tušek Žnidarič, M.; Mozetič Vodopivec, B.; Smole Možina, S. Anti-Campylobacter activity of resveratrol and an extract from waste Pinot noir grape skins and seeds, and resistance of Camp. jejuni planktonic and biofilm cells, mediated via the Cme ABC efflux pump. J. Appl. Microbiol. 2017, 122, 65-77. [CrossRef]

31. Duarte, A.; Alves, A.C.; Ferreira, S.; Silva, F.; Domingues, F.C. Resveratrol inclusion complexes: Antibacterial and anti-biofilm activity against Campylobacter spp. and Arcobacter butzleri. Food Res. Int. 2015, 77, 244-250. [CrossRef]

32. Baur, J.A.; Sinclair, D.A. Therapeutic potential of resveratrol: The in vivo evidence. Nat. Rev. Drug Discov. 2006, 5, 493-506. [CrossRef]

33. Bode, L.M.; Bunzel, D.; Huch, M.; Cho, G.S.; Ruhland, D.; Bunzel, M.; Bub, A.; Franz, C.M.; Kulling, S.E. In vivo and in vitro metabolism of trans-resveratrol by human gut microbiota. Am. J. Clin. Nutr. 2013, 97, 295-309. [CrossRef]

34. Zhou, Z.X.; Mou, S.F.; Chen, X.Q.; Gong, L.L.; Ge, W.S. Anti-inflammatory activity of resveratrol prevents inflammation by inhibiting NF-кB in animal models of acute pharyngitis. Mol. Med. Rep. 2018, 17, 1269-1274. [CrossRef] [PubMed]

35. Schwager, J.; Richard, N.; Widmer, F.; Raederstorff, D. Resveratrol distinctively modulates the inflammatory profiles of immune and endothelial cells. BMC Complement. Altern. Med. 2017, 17, 309. [CrossRef] [PubMed]

36. Murray, P.J.; Allen, J.E.; Biswas, S.K.; Fisher, E.A.; Gilroy, D.W.; Goerdt, S.; Gordon, S.; Hamilton, J.A.; Ivashkiv, L.B.; Lawrence, T.; et al. Macrophage activation and polarization: Nomenclature and experimental guidelines. Immunity 2014, 41, 14-20. [CrossRef] [PubMed]

37. Gao, X.; Xu, Y.X.; Janakiraman, N.; Chapman, R.A.; Gautam, S.C. Immunomodulatory activity of resveratrol: Suppression of lymphocyte proliferation, development of cell-mediated cytotoxicity, and cytokine production. Biochem. Pharmacol. 2001, 62, 1299-1308. [CrossRef]

38. Švajger, U.; Jeras, M. Anti-inflammatory effects of resveratrol and its potential use in therapy of immune-mediated diseases. Int. Rev. Immunol. 2012, 31, 202-222. [CrossRef] 
39. Bereswill, S.; Munoz, M.; Fischer, A.; Plickert, R.; Haag, L.M.; Otto, B.; Kuhl, A.A.; Loddenkemper, C.; Gobel, U.B.; Heimesaat, M.M. Anti-inflammatory effects of resveratrol, curcumin and simvastatin in acute small intestinal inflammation. PLoS ONE 2010, 5, e15099. [CrossRef] [PubMed]

40. Wang, Z.L.; Luo, X.F.; Li, M.T.; Xu, D.; Zhou, S.; Chen, H.Z.; Gao, N.; Chen, Z.; Zhang, L.L.; Zeng, X.F. Resveratrol possesses protective effects in a pristane-induced lupus mouse model. PLoS ONE 2014, 9, e114792. [CrossRef]

41. Voloshyna, I.; Hai, O.; Littlefield, M.J.; Carsons, S.; Reiss, A.B. Resveratrol mediates anti-atherogenic effects on cholesterol flux in human macrophages and endothelium via PPAR $\gamma$ and adenosine. Eur. J. Pharmacol. 2013, 698, 299-309. [CrossRef]

42. Riba, A.; Deres, L.; Sumegi, B.; Toth, K.; Szabados, E.; Halmosi, R. Cardioprotective Effect of Resveratrol in a Postinfarction Heart Failure Model. Oxid. Med. Cell Longev. 2017, 2017, 6819281. [CrossRef]

43. Zhang, C.; Zhao, X.H.; Yang, L.; Chen, X.Y.; Jiang, R.S.; Jin, S.H.; Geng, Z.Y. Resveratrol alleviates heat stress-induced impairment of intestinal morphology, microflora, and barrier integrity in broilers. Poult. Sci. 2017, 96, 4325-4332. [CrossRef]

44. Den Hartogh, D.J.; Tsiani, E. Health Benefits of Resveratrol in Kidney Disease: Evidence from In Vitro and In Vivo Studies. Nutrients 2019, 11, 1624. [CrossRef] [PubMed]

45. Aktaş, H.S.; Ozel, Y.; Ahmad, S.; Pençe, H.H.; Ayaz-Adakul, B.; Kudas, I.; Tetik, S.; Şekerler, T.; Canbey-Göret, C.; Kabasakal, L.; et al. Protective effects of resveratrol on hepatic ischemia reperfusion injury in streptozotocininduced diabetic rats. Mol. Cell Biochem. 2019, 460, 217-224. [CrossRef] [PubMed]

46. Heebøll, S.; Thomsen, K.L.; Pedersen, S.B.; Vilstrup, H.; George, J.; Grønbæk, H. Effects of resveratrol in experimental and clinical non-alcoholic fatty liver disease. World J. Hepatol. 2014, 6, 188-198. [CrossRef] [PubMed]

47. Wu, B.; Xiao, Z.; Zhang, W.; Chen, H.; Liu, H.; Pan, J.; Cai, X.; Liang, G.; Zhou, B.; Shan, X.; et al. A novel resveratrol-curcumin hybrid, a19, attenuates high fat diet-induced nonalcoholic fatty liver disease. Biomed. Pharmacother. 2019, 110, 951-960. [CrossRef] [PubMed]

48. Kløve, S.; Genger, C.; Mousavi, S.; Weschka, D.; Bereswill, S.; Heimesaat, M.M. Toll-Like Receptor-4 Dependent Intestinal and Systemic Sequelae Following Peroral Campylobacter coli Infection of IL10 Deficient Mice Harboring a Human Gut Microbiota. Pathogens 2020, 9, 386. [CrossRef]

49. Youn, H.S.; Lee, J.Y.; Fitzgerald, K.A.; Young, H.A.; Akira, S.; Hwang, D.H. Specific inhibition of MyD88-independent signaling pathways of TLR3 and TLR4 by resveratrol: Molecular targets are TBK1 and RIP1 in TRIF complex. J. Immunol. 2005, 175, 3339-3346. [CrossRef]

50. Chen, J.; Cao, X.; Cui, Y.; Zeng, G.; Chen, J.; Zhang, G. Resveratrol alleviates lysophosphatidylcholine-induced damage and inflammation in vascular endothelial cells. Mol. Med. Rep. 2018, 17, 4011-4018. [CrossRef]

51. Tomé-Carneiro, J.; Gonzálvez, M.; Larrosa, M.; Yáñez-Gascón, M.J.; García-Almagro, F.J.; Ruiz-Ros, J.A.; Tomás-Barberán, F.A.; García-Conesa, M.T.; Espín, J.C. Grape resveratrol increases serum adiponectin and downregulates inflammatory genes in peripheral blood mononuclear cells: A triple-blind, placebo-controlled, one-year clinical trial in patients with stable coronary artery disease. Cardiovasc. Drugs Ther. 2013, 27, 37-48. [CrossRef]

52. Patel, K.R.; Scott, E.; Brown, V.A.; Gescher, A.J.; Steward, W.P.; Brown, K. Clinical trials of resveratrol. Ann. N. Y. Acad. Sci. 2011, 1215, 161-169. [CrossRef]

Publisher's Note: MDPI stays neutral with regard to jurisdictional claims in published maps and institutional affiliations.

(C) 2020 by the authors. Licensee MDPI, Basel, Switzerland. This article is an open access article distributed under the terms and conditions of the Creative Commons Attribution (CC BY) license (http://creativecommons.org/licenses/by/4.0/). 